\section{EFICÁCIA DA PROMOÇÃO DA SAÚDE ORAL NO CONTROLO DO BIOFILME BUCAL}

\author{
Oral health promotion efficiency in the control of oral biofilm \\ Eficácia de la promoción de la salud oral en el control del \\ biofilm dental
}

\section{RESUMO}

Objetivo: Avaliar a eficácia das instruções de higiene oral, em particular no controlo do biofilme dentário, bem como avaliar a eficácia do reforço dessas instruções. Métodos: Realizou-se estudo quasi-experimental na Clínica Universitária da Universidade Católica Portuguesa (UCP) em Viseu-Portugal, desenvolvido entre janeiro e junho de 2012. Efectuouse três avaliações a 30 indivíduos através do Índice de Placa de O'Leary e aplicação de questionário abordando o comportamento de saúde oral, com um mês de periodicidade entre cada medição. Na primeira avaliação, todos os indivíduos receberam as mesmas instruções de higiene oral, registo do Índice de Placa de O’Leary e aplicação do questionário. Na segunda avaliação, realizou-se um novo registo do índice a todos os indivíduos, mas apenas o grupo de intervenção $(n=16)$ recebeu o reforço dessas instruções e na terceira avaliação executouse um novo registo a todos os indivíduos $(n=30)$. Resultados: Tanto o grupo de intervenção como o controlo apresentaram uma redução do índice, mas aquele mostrou uma redução mais importante na última avaliação realizada quando comparada a este: $38,19 \%(\mathrm{n}=16)$ vs. $69,57 \%(\mathrm{n}=14)$, respectivamente $(\mathrm{p}<0,05)$. Relativamente à frequência de escovação dentária, no grupo de intervenção, $68,8 \%(\mathrm{n}=11)$ escovavam pelo menos duas vezes por dia, enquanto no grupo de controlo apenas $57,1 \%(n=8)$ escovavam com a mesma frequência. Nesse caso, foram registadas diferenças significativas entre os dois grupos $(p<0.05)$. Conclusão: A promoção da saúde oral por meio de instruções de higiene demonstrou ser eficaz na melhoria dos comportamentos de saúde e, consequentemente, no controlo do biofilme dentário.

Descritores: Biofilmes; Escovação Dentária; Motivação; Educação.

\section{ABSTRACT}

Objective: To assess the efficacy of oral health instructions, particularly in the control of dental plaque, as well as assess the effectiveness of the reinforcement of the same instructions. Methods: A quasi-experimental study was conducted at the University Clinic of the Portuguese Catholic University (UCP) in Viseu-Portugal from January to June 2012. Three different assessments were performed with 30 participants using the O'Leary Plaque Index and a questionnaire on oral health behavior, with a one-month interval between each assessment. In the first assessment, all participants received the same instructions of oral hygiene and the O'Leary Plaque Index registration and the application of the questionnaire were performed; in the second assessment, a new registration of the O'Leary Index was made, but only the experimental group $(n=16)$ received the reinforcement of oral health instructions, and in the third assessment, a new registration of the O'Leary Plaque Index was made for all the individuals $(n=30)$. Results: Both control and experimental groups showed a decrease in the O'Leary Plaque Index, but the latter showed a more significant decrease in the last assessment: $38.19 \%(n=16) v s .69 .57 \%(n=14), p<0.05$. Regarding the frequency of toothbrushing, in the experimental group, $68.8 \%(n=11)$ brushed the teeth at least twice a day, while in the control group only $57.1 \%(n=8)$ performed the same frequency of toothbrushing. In this case, statistically significant differences were found between the two groups $(p<0.05)$. Conclusion: Oral health promotion through oral hygiene instruction was effective in improving oral health behaviors, and, consequentely, the control of dental biofilm.

Descriptors: Biofilms; Toothbrushing; Motivation; Education.
Artigo Original
1) Departamento de Ciências da Saúde Universidade Católica Portuguesa (UCP) - Viseu - Portugal

2) Unidade de Saúde Familiar Grão Vasco Viseu - Portugal
Recebido em: 07/04/2013

Revisado em: 05/06/2013 Aceito em: 10/09/2013 


\section{RESUMEN}

Objetivo: Evaluar la eficacia de las instrucciones de higiene oral, en especial del control del biofilm dental así como evaluar la eficacia del refuerzo de dichas instrucciones. Métodos: Se realizó un estudio cuasi-experimental en la Clínica Universitaria de la Universidad Católica Portuguesa (UCP) de ViseuPortugal, desarrollado entre enero y junio de 2012. Se realizó tres evaluaciones en 30 individuos a través del Índice de Placa de O'Leary y la aplicación del cuestionario incluyendo la conducta de salud oral con un mes de intervalo para cada medición. En la primera evaluación todos los individuos recibieron las mismas instrucciones de higiene oral, registro del Índice de Placa de O'Leary y la aplicación del cuestionario. En la segunda evaluación se realizó un nuevo registro del índice en todos los individuos pero solamente el grupo de intervención $(n=16)$ recibió el refuerzo de esas instrucciones y en la tercera evaluación se hizo un nuevo registro de todos los individuos $(n=30)$. Resultados: $E l$ grupo de intervención y el grupo control presentaron reducción del índice, pero el grupo de intervención mostro una reducción más importante en la última evaluación realizada al compararse al grupo control: el 38,19\% (n=16) vs. el 69,57\% (n=14), respectivamente $(p<0,05)$. Respecto la frecuencia de cepillado dentario, en el grupo de intervención el 68,8\% $(n=11)$ cepillaban al menos dos veces al día mientras que en el grupo control solamente el 57,1\% (n=8) cepillaban con la misma frecuencia. En ese caso, fueron registradas diferencias significativas entre los grupos ( $<<0.05)$. Conclusión: La promoción de la salud oral a través de instrucciones de higiene ha sido eficaz para la mejoría de conductas en salud $y$, por consiguiente, para el control del biofilm dental.

Descriptores: Biofilmes; Cepillado Dental; Motivación; Educación.

\section{INTRODUÇÃO}

O organismo está sujeito a colonizações bacterianas ao longo da vida, mas normalmente existe uma coexistência pacífica. Na cavidade oral, os dentes proporcionam uma superfície de colonização não descamativa, permitindo o desenvolvimento de depósitos bacterianos ${ }^{(1,2)}$. Esses depósitos bacterianos permitem a formação do biofilme dentário que consiste no desenvolvimento de diversas fases até ao estabelecimento do biofilme maturo em menos de 24 horas. A não remoção desta implica o desenvolvimento de diferentes patologias orais ${ }^{(3,4)}$.

Existem diferentes metodologias de quantificação do biofilme dentário, contudo, as mais utilizadas são aquelas que recorrem a agentes reveladores, uma vez que permitem a educação e motivação do paciente para melhorar a eficiência dos procedimentos de controlo do biofilme dentário $^{(4)}$. No entanto, deve-se considerar que a quantidade do biofilme não equaciona a severidade da doença, pelo que, actualmente, os índices de biofilme dentário são aplicados como medida de avaliação da eficácia da higiene oral ${ }^{(5)}$.

Estudos epidemiológicos evidenciam a associação entre higiene oral e a formação do biofilme dentário, sendo que o controlo mecânico é reconhecido como o meio mais simples e com melhor relação custo/benefício ${ }^{(6-11)}$. O controlo mecânico do biofilme dentário é um importante meio de manutenção das condições de saúde oral, evitando ou retardando a colonização do meio gengival por espécies bacterianas ${ }^{(6)}$. Entretanto, preconiza-se que um aspecto inerente ao sucesso desse controlo é a motivação criada no paciente ${ }^{(5)}$. A escovação e o uso de meios adicionais de remoção mecânica do biofilme, como o fio dental ou a escova interdental, permitem o controlo adequado quando devidamente utilizados. No entanto, o uso frequente da escova não é sinónimo de higienização, pois mais importante que a quantidade é a qualidade da técnica de escovação( ${ }^{(7)}$.

Cabe ainda ao profissional educar e motivar o paciente $^{(12)}$. A educação tem como objectivo principal motivar para uma mudança de atitude do paciente em relação a hábitos de higiene oral, que é alcançada através da criação ou mudança da percepção por parte do paciente. Para que se alcance essa mudança, é fundamental a motivação continuada do paciente ${ }^{(8,12)}$.

A educação para a promoção da saúde oral é um aspecto muito importante para a saúde pública. Assim, este estudo buscou demonstrar como o reforço da educação para a saúde oral interfere no controlo das doenças orais na população. Apesar de se tratar de uma área de extrema importância, uma vez que visa a prevenção primária na população, os estudos são escassos e limitados, sendo que o desenvolvimento de novas pesquisas é essencial para o acréscimo de conhecimento científico nesse campo.

Assim, definiu-se como objectivo geral deste estudo avaliar a eficácia das instruções de higiene oral, em particular no controlo do biofilme dentário, bem como a eficácia do reforço dessas mesmas instruções.

\section{MÉTODOS}

Realizou-se um estudo quasi-experimental que consistiu na avaliação de pacientes odontológicos voluntários consultados na Clínica Universitária da Universidade Católica Portuguesa (UCP), Viseu, Portugal, desenvolvido entre janeiro e junho de 2012. Os estudos quasiexperimentais são estudos em que existe manipulação da intervenção, mas não atribuição aleatória desta. São exemplos de estudos quasi-experimentais certos ensaios de campo, certos ensaios de intervenções na comunidade e os ensaios clínicos não randomizados ${ }^{(13)}$. 
Foram considerados os seguintes critérios de inclusão: pacientes que recorreram à Clínica Universitária da UCP pela primeira vez e com dentição permanente não considerando a presença ou ausência dos terceiros molares. Os critérios de exclusão considerados para a execução do estudo foram: pacientes totalmente edêntulos ou portadores de aparelho ortodôntico fixo, bem como aqueles que não comparecessem nas reavaliações.

A amostra inicial do presente estudo consistia em 40 indivíduos que afirmaram inicialmente querer participar no estudo, sendo 18 indivíduos do género feminino $(45 \%)$ e 22 do género masculino (55\%). A amostragem obtida foi por conveniência, pois participaram os indivíduos que frequentavam a consulta de Medicina Dentária Preventiva na Clínica Universitária da UCP. Dos 40 indivíduos como amostra inicial, 10 foram excluídos por não comparecerem às reavaliações, levando a uma amostra de 30 indivíduos, dos quais 13 eram do género feminino (43\%) e 17 , do género masculino (57\%).

Constituíram-se dois grupos para esta investigação, sem alocação aleatória: um grupo de controlo com 14 indivíduos (sem reforço das instruções de higiene oral) e um grupo de intervenção educativa com 16 indivíduos (com reforço das instruções de higiene oral).

A recolha de dados foi efectuada em 3 períodos distintos. A primeira recolha compreendeu a avaliação do Índice de Placa de O'Leary inicial, instruções de higiene oral e motivação de ambos os grupos e preenchimento de um questionário sobre comportamentos de saúde oral elaborado pelos pesquisadores responsáveis pelo estudo.

A segunda consistiu na reavaliação do Índice de Placa de O'Leary um mês depois para ambos os grupos, havendo o reforço das instruções de higiene oral e motivação apenas para o grupo de intervenção. A terceira incluiu a avaliação do Índice de Placa de O’Leary dois meses depois da primeira avaliação e preenchimento do questionário para ambos os grupos.

De forma a reduzir o viés na recolha de dados, esta foi realizada por uma única operadora, que efectuou a observação intraoral e o registro das observações realizadas, auxiliou os participantes no preenchimento do questionário e nas instruções de higiene oral e motivação para comportamentos de saúde oral adequados.

O protocolo de intervenção consistiu: 1) na breve explicação e entrega do consentimento informado ao paciente; 2) aplicação de revelador de placa (eritrosina 2\%) em todas as superfícies dentárias; 3 ) solicitação do paciente para que bocheche de forma a remover os excessos do revelador de biofilme dentário; 4) avaliação das quatro faces de cada dente (mesial, distal, vestibular, lingual/palatino) e anotação no diagrama. Essa observação era realizada em ambiente clínico, o que permitiu a utilização do candeeiro usualmente encontrado num consultório médico-dentário com boa iluminação; 5) determinação do Índice de Placa de O'Leary; 6) recurso a espelho de mão para educação do paciente seguido de instruções de higiene oral; 7) preenchimento do questionário por parte do paciente; e 8) instrução para nova avaliação após 1 mês.

Neste estudo, a variável quantitativa limitou-se à determinação do Índice de Placa de O'Leary. Este preconiza a aplicação do revelador nas superfícies supragengivais dos dentes e a avaliação das quatro faces dentárias (vestibular, mesial, distal, palatina/lingual). Após o paciente bochechar para remover o excesso de revelador, cada superfície, exceptuando a face oclusal, é avaliada e registada segundo a ausência/presença de coloração no diagrama. Após cada superfície ser pontuada, calcula-se o índice dividindo o número de faces pintadas pelo número de faces totais, convertendo o valor obtido em percentagem ${ }^{(5)}$.

Como variáveis qualitativas, avaliou-se os comportamentos de saúde oral através de um questionário aplicado em períodos avaliativos diferentes. O primeiro no início do estudo e o segundo no final deste. Essa caracterização baseou-se em questões referentes aos cuidados de higiene oral, como o número de escovagens diárias e utilização de fio dentário.

No processamento e análise de dados, utilizou-se o Statistical Package for the Social Sciences (SPSS 18.0). A descrição das variáveis contínuas foi efectuada através de medidas de tendência central (média) e as prevalências expressas em percentagens. Para comparação entre o grupo de intervenção e o grupo controlo, utilizou-se o teste $\mathrm{T}$ para amostras independentes e o ANOVA para comparação intragrupo.

Para a realização deste estudo, solicitou-se a aprovação por parte do Departamento de Ciências da Saúde da UCP, Viseu, Portugal. O pedido de autorização para a realização do estudo foi efectuado por escrito aos participantes. A informação recolhida por questionário era anónima, voluntária e confidencial, não havendo qualquer tipo de referência nominal aos participantes.

\section{RESULTADOS}

Detectou-se uma diminuição da média das percentagens do Índice de Placa de O'Leary ao comparar a avaliação inicial com a realizada após o primeiro mês e o segundo mês, como indica a Tabela I. 
Tabela I - Registo dos Índices de Placa de O’Leary (IP) na avaliação inicial (IP1), após 1 mês (IP2) e após 2 meses (IP3). Viseu-Portugal, 2012.

\begin{tabular}{lccc}
\hline Índice de Placa & Média (\%) & Desvio Padrão & p\# \\
\hline IP1 & 74,00 & 21,17 & \\
IP2 & 63,79 & 24,92 & $<0,001$ \\
IP3 & 52,83 & 25,44 & $<0,019$ \\
\hline
\end{tabular}

\#em comparação à avaliação inicial (IP1)

Relativamente ao grupo de intervenção, verificou-se uma diminuição gradual do percentual do Índice de Placa de O'Leary, enquanto no grupo de controlo houve um ligeiro aumento da percentagem da primeira para a segunda avaliação (Tabela II). No grupo de intervenção, a média dos valores obtidos no registo do Índice de Placa de O'Leary, para o Índice de Placa 1, foi 75\%; para o Índice de Placa 2 (1 mês), foi 53,88\%; e para o Índice de Placa 3 (2 meses), foi $38,50 \%$. Verificou-se neste grupo a existência de diferenças estatisticamente significativas entre os registos do Índice de Placa de O'Leary realizados $(\mathrm{p}<0,05)$.

No grupo de controlo, a média dos valores obtidos no registo do Índice de placa de O'Leary (Tabela II) foram: para o Índice de Placa 1 ( 0 meses), 72,89\%; para o Índice de Placa 2 (1 mês), 74,31\%; e para o Índice de Placa 3 (2 meses), 69,57\%. Entretanto, nesse grupo, não houve diferenças estatisticamente significativas entre os registos do Índice de Placa de O'Leary realizados ( $>00,05)$.

Tabela II - Registo dos Índices de Placa de O’Leary (IP) na avaliação inicial (IP1), após 1 mês (IP2) e após 2 meses (IP3) nos grupos de intervenção e controlo. Viseu-Portugal, 2012.

\begin{tabular}{lccc}
\hline Índice de Placa & Média (\%) & Desvio Padrão & $p \#$ \\
\hline Grupo de Intervenção & & & \\
IP1 & 75,00 & 21,18 & $<0,001$ \\
IP2 & 53,88 & 28,10 & $<0,002$ \\
IP3 & 38,19 & 17,84 & \\
Grupo Controlo & & & $>0,1$ \\
IP1 & 72,89 & 21,69 & $>0,05$ \\
IP2 & 74,31 & 15,91 & \\
IP3 & 69,57 & 22,60 & \\
\hline
\end{tabular}

\#em comparação à avaliação inicial (IP1)

Tabela III - Registo dos Índices de Placa de O'Leary (IP) na avaliação inicial (IP1), após 1 mês (IP2) e após 2 meses (IP3) em relação a variável género. Viseu-Portugal, 2012.

\begin{tabular}{lccc}
\hline Índice de Placa & Média (\%) & Desvio Padrão & p\# \\
\hline Género feminino & & & \\
IP1 & 71,83 & 23,79 & 0,002 \\
IP2 & 62,57 & 21,18 & 0,013 \\
IP3 & 49,62 & 25,63 & \\
Género masculino & & & \\
IP1 & 75,77 & 19,16 & 0,009 \\
IP2 & 64,68 & 23,84 & 0,018 \\
IP3 & 55,29 & 25,79 & \\
\hline
\end{tabular}

\#em comparação à avaliação inicial (IP1) 
Nos indivíduos de género feminino, a média dos valores obtidos no registo do Índice de Placa de O'Leary (Tabela III) foi: para o Índice de Placa 1 (avaliação inicial), 71,83\%; para o Índice de Placa 2 (1 mês), 62,57\%; e para o Índice de Placa 3 (2 meses), 49,62\%. Nesse caso, verificouse diferenças significativas entre IP1 e IP3, bem como entre IP2 e IP3.

Nos indivíduos de género masculino, a média dos valores obtidos no registo do Índice de Placa de O'Leary (Tabela IV) foram: para o Índice de Placa 1 (0 meses), 75,77\%; para o Índice de Placa 2 ( 1 mês), 64,68\%; e para o Índice de Placa 3 (2 meses), 55,29\%. Na comparação dos três registos para o género masculino, também verificámos que existem diferenças estatisticamente significativas entre IP1 e IP3 e entre IP2 e IP3 $(\mathrm{p}<0,05)$.

Relativamente ao número de escovagens diárias iniciais, $80 \%(\mathrm{n}=32)$ dos indivíduos escovavam os dentes uma ou duas vezes por dia. A frequência estimada neste estudo foi superior entre os indivíduos do género feminino $(77,7 \%, n=14)$. Verificou-se também que apenas $10 \%(n=4)$ dos indivíduos não efectuavam nenhuma escovação diária, sendo $13,6 \%(\mathrm{n}=3)$ do género masculino.

Quando comparados os resultados obtidos no final do estudo, todos os indivíduos passaram a efectuar pelo menos uma escovação diária, um aumento significativo de indivíduos passaram a efectuar duas escovações por dia $(63,3 \%, \mathrm{n}=19)$, bem como um aumento de indivíduos a realizar três escovações diárias $(20 \%, \mathrm{n}=6)$. Contudo, verificou-se no grupo de intervenção que $68,8 \%(n=11)$ escovavam pelo menos duas vezes por dia, enquanto no grupo de controlo apenas $57,1 \%(n=8)$ escovavam com a mesma frequência. Nesse caso, foram registados diferenças estatisticamente significativas entre os dois grupos $(\mathrm{p}=0.03)$.

Quanto à utilização do fio dental, verificou-se que apenas $30 \%(\mathrm{n}=12)$ dos indivíduos questionados utilizavam fio dentário, sendo que os indivíduos do género feminino recorriam em maior número $(44,4 \%, n=8)$ a esse meio auxiliar de controlo mecânico da placa bacteriana que os indivíduos do género masculino $(18,2 \%, \mathrm{n}=4)$. Comparando os resultados finais, foi observável um aumento significativo de indivíduos a usarem fio dentário no grupo de intervenção $(75,0 \%, \mathrm{n}=12)$, comparativamente aos do grupo de controlo $(29,0 \%, n=4)(p=0,02)$.

\section{DISCUSSÃO}

No que diz respeito aos comportamentos de saúde oral, verificou-se no presente estudo que os comportamentos de saúde oral iniciais na amostra estudada são débeis, podendo, no entanto, ser alterados por meio da promoção da saúde oral, através das instruções de higiene oral e motivação. Os resultados finais obtidos indicam uma melhoria generalizada dos comportamentos de saúde oral, verificando-se benefícios acentuados no grupo de intervenção face ao grupo de controlo.

Esses resultados vão de encontro a estudos segundo os quais o reforço da educação e a instrução continuada de bons hábitos de higiene oral permite a melhoria da saúde oral e o aumento da frequência e motivação para a realização da escovação ${ }^{(14,12)}$.

Nesse caso, não deixa de ser importante referir a baixa frequência de utilização diária do fio dental por parte dos participantes do presente estudo, o que vai de encontro aos resultados de outros estudos também realizados em Portugal ${ }^{(15,16)}$.

Quando analisado o Índice de Placa de O’Leary, verificamos que os resultados percentuais demonstram uma melhoria da média do Índice de Placa de O'Leary no total dos indivíduos da amostra, diminuindo de um Índice de Placa inicial de 74\% para um Índice de Placa final de $52,83 \%$. O resultado vai de encontro aos obtidos em estudo brasileiro com 135 escolares da rede estadual e municipal de um município do Rio Grande do Sul. Neste estudo, avaliou-se a eficácia de duas estratégias motivacionais em relação ao controlo do biofilme dentário e hemorragia gengival. Uma implicava uma sessão de motivação única (grupo A) e outra motivação em várias sessões (grupo B), sendo que para ambos os grupos houve redução dos índices estudados ${ }^{(17)}$.

No presente estudo, os indivíduos de género feminino apresentam um Índice de Placa inicial mais baixo face aos indivíduos do género masculino o que revelou uma melhoria nos hábitos de higiene oral. A obtenção desses resultados apenas vem confirmar o maior cuidado com a higiene oral apresentado no género feminino, comparativamente ao género masculino ${ }^{(18,19)}$.

Quando comparados os grupos estudados, verificouse uma melhoria significativa no grupo de intervenção de um Índice de Placa inicial de 75\% para um Índice de Placa final de $38,19 \%$, o que pode ser justificado pela aplicação mais constante e frequente ao nível da educação para a saúde oral que o grupo de intervenção foi sujeita durante o estudo, adquirindo melhores hábitos de higiene oral comparativamente aos registados no grupo de controlo $^{(17,20-22)}$. No presente estudo, o grupo de controlo apresentou uma melhoria, embora substancialmente menos significativa - de um Índice de Placa inicial de 72,89\% para um Índice de Placa final de 69,57\%. Nesse grupo, verificouse que, da primeira para a segunda avaliação, houve um aumento dos valores de Índice de Placa (IP2=74,31\%).

Historicamente, detectam-se os benefícios de programa dentário e motivação continuada para controle do biofilme 
dentário. Estudo realizado na décade de 1990, num grupo de controlo e experimental recorrendo ao Índice de Placa de O'Leary, concluiu que o programa de motivação continuada diminuiu significativamente o Índice de Placa na amostra pertencente ao grupo experimental ${ }^{(22)}$.

Com relação à importância do reforço contínuo das instruções de higiene oral, observa-se que grupos com sessões múltiplas de motivação apresentam uma redução significativa do biofilme dental e hemorragias gengivais, quando comparados com os grupos de sessão única ${ }^{(17)}$.

Os benefícios verificados com o reforço das instruções vieram corroborar com trabalhos anteriores, aspecto pelo qual deve ser encarado como ferramenta a ser utilizada pelo cirurgião-dentista nas consultas, conduzindo à melhoria na qualidade da saúde oral da comunidade ${ }^{(23)}$.

Reconhecem-se algumas limitações inerentes a este estudo, nomeadamente, o reduzido período de tempo disponível, que levou a uma amostra reduzida, impedindo a extrapolação dos resultados para a população-alvo. Outra limitação consistiu na dependência da comparência dos indivíduos às três avaliações em momentos específicos. $\mathrm{O}$ não cumprimento implicava a exclusão do estudo, estando, assim, a amostra dependente de uma variável não controlada.

\section{CONCLUSÕES}

A promoção da saúde oral por meio da educação e motivação demonstrou ser eficaz na melhoria dos comportamentos de saúde e, consequentemente, no controlo do biofilme dentário. Perante essa relação positiva, verificou-se uma exacerbação da mesma aquando aplicado o reforço dessas mesmas instruções.

Através de um programa de promoção de saúde oral, os comportamentos de saúde oral são susceptíveis a mudanças positivas, mas, ao longo do período de ensaio, verificou-se uma melhoria nos dois géneros, embora mais acentuada nos indivíduos do género feminino.

\section{REFERÊNCIAS}

1. Listgarten M, Korostoff J. Cultural Health Influences. In: Harris NO, García-Godoy F. Primary Preventive Dentistry. $8^{\text {th }}$ ed. New Jersey: Pearson Prentice Hall; 2013. p. 23-44.

2. Maltz M, Jardim J, Alves L. Health promotion and dental caries. Braz Oral Res. 2010;24(Supl 1):18-25.

3. Ramage G, Culshaw S, Jones B, Williams C. Are we any closer to beating the biofilm: novel methods of biofilm control. Curr Opin Infect Dis. 2010;23(6):560-6.
4. Newman M, Takei H, Klokkevold P, Carranza F. Carranza's Clinical Periodontology. $11^{\text {th }}$ ed. St Louis: Elvisier Saunders; 2012.

5. Darby M. Mosby's comprehensive review of dental hygiene. $7^{\text {th }}$ ed. St Louis: Elsevier Mosby; 2012.

6. Dutra CMR, Ferreira EF. A motivação de pacientes portadores de doença periodontal crônica sob manutenção periodontal: um estudo qualitativo. Rev Odontol UNESP. 2005;34(1):5-10.

7. World Health Organization - WHO, Kaban Moslehzadeh. Oral hygiene indices [acesso em 2010 Fev 20]. Disponível em: http://www.whocollab. od.mah.se/expl/ohiintrod.html

8. Nightingale K, Chinta S, Agarwal P, Nemelivsky M, Frisina A, Cao Z, et al. Toothbrush efficacy for plaque removal. Int J Dent Hyg. 2014;14:1-7.

9. Casanova-Rosado JF, Vallejos-Sánchez AA, Minaya-Sánchez M, Medina-Solís CE, De La RosaSantillana R, Márquez-Corona ML, et al. Frequency of tooth brushing and associated factors in Mexican schoolchildren six to nine years of age. West Indian Med J. 2013;62(1):68-72.

10. Navarro RS, Esteves GV, Youssef MN. Estudo clínico do comportamento de escolares mediante escovação supervisionada e motivação no controle de placa bacteriana. Rev Odontol Univ São Paulo. 1996;10(2):153-7.

11. Cury JA, Tenuta LM. Evidence-based recommendation on toothpaste use. Braz Oral Res. 2014;28(Spe 1):1-7.

12. Bardal P, Olympio K, Bastos J, Henriques J, Buzalaf $\mathrm{M}$. Education and motivation in oral health - preventing disease and promoting health in patients undergoing orthodontic treatment. Dental Press J Orthod. 2011;16(3):95-102.

13. Gordis L. Epidemiology. 5th ed. Philadelphia: Elvesier Saunders; 2014.

14. Hurling R, Claessen JP, Nicholson J, Schäfer F, Tomlin CC, Lowe CF. Automated coaching to help parents increase their children's brushing frequency: an exploratory trial. Community Dent Health. 2013;30(2):88-93.

15. Pereira C, Veiga N, Amaral O, Pereira J. Comportamentos de saúde oral em adolescentes portugueses. Rev Port Saúde Pública. 2013;31(2):15865.

16. Barata C, Veiga N, Mendes C, Araujo F, Ribeiro O, Coelho I. Determinação do CPOD e comportamentos 
de saúde oral numa amostra de adolescentes do concelho de Mangualde. Rev Port Estomatol Med Dent Cir Maxilo Fac. 2013;54(1):27-32.

17. Toassi RFC, Petry PC. Motivação no controle do biofilme dental e sangramento gengival em escolares. Rev Saúde Pública. 2002;36(5):634-7.

18. Bastos RS, Carvalho ES, Xavier A, Caldana ML, Bastos J, Lauris J. Dental caries related to quality if life in two Brazilian adolescente groups: a cross-sectional randomised study. Int Dent J. 2012;62(3):137-43.

19. Poutanen R, Lahti S, Seppa L, Tolvanen M, Hausen H. Oral health-related knowledge, attitudes, behavior, and family characteristics among Finnish schoolchildren with and without active initial caries lesion. Acta Odontol Scand. 2007;65(2):87-96.

20. Marini I, Bortolotti F, Parenti SI, Gatto MR, Bonetti GA. Combined effects of repeated oral hygiene motivation and type of toothbrush on orthodontic patients: A blind randomized clinical trial. Angle Orthod. 2014;84(5):896-901.
21. Haleem A, Siddiqui M, Khan A. School-based strategies for oral health education of adolescents - a cluster randomized controlled trial. BMC Oral Health. 2012;12(542):1-12.

22. Pompeu JGF. Avaliação da eficácia de programa odontológico de motivação continuada para estabelecimento e manutenção da higiene bucal. Camaragibe; 1997.

23. Kumar S, Konde S, Raj S, Agarwal M. Effect of oral health education and fluoridated dentifrices on the oral health status of visually impaired children. Contemp Clin Dent. 2012;3(4):398-401.

\section{Endereço para correspondência:}

Nélio J. Veiga

Departamento de Ciências da Saúde - Universidade

Católica Portuguesa

Estrada da Circunvalação, 3504-505

Viseu - Portugal

E-mail: nelioveiga@gmail.com 